\title{
Availability of ambulance patient care reports in the emergency department
}

\author{
Dominick Shelton, Paul Sinclair \\ Sunnybrook Health Sciences Centre, Toronto, Canada
}

\begin{abstract}
Clinical handovers of patient care among healthcare professionals is vulnerable to the loss of important clinical information. A verbal report is typically provided by paramedics and documented by emergency department (ED) triage nurses. Paramedics subsequently complete a patient care report which is submitted electronically. This emergency medical system (EMS) patient care report often contains details of paramedic assessment and management that is not all captured in the nursing triage note. EMS patient care reports are often unavailable for review by emergency physicians and nurses.
\end{abstract}

Two processes occur in the distribution of EMS patient care reports. The first is an external process to the ED that is influenced by the prehospital emergency medical system and results in the report being faxed to the ED. The second process is internal to the ED that requires clerical staff to distribute the fax report to accompany patient charts.

A baseline audit measured the percentage of EMS patient care reports that were available to emergency physicians at the time of initial patient assessments and showed a wide variation in the availability of EMS reports. Also measured were the time intervals from patient transfer from EMS to ED stretcher until the EMS report was received by fax (external process measure) and the time from receiving the EMS fax report until distribution to patient chart (internal process measure). These baseline measures showed a wide variation in the time it takes to receive the EMS reports by fax and to distribute reports.

Improvement strategies consisted of:

1. Educating ED clerical staff about the importance of EMS reports

2. Implementing a new process to minimize ED clerical staff handling of EMS reports for nonactive ED patients

3. Elimination of the automatic retrieval of old hospital charts and their distribution for ED patients

4. Introduction of an electronic dashboard for patients arriving by ambulance to facilitate more efficient distribution of EMS reports.

Implementation of change strategies did not result in a significant improvement in the percentage of EMS reports available to emergency physicians at the time of initial patient assessment. However, tracking both external and internal processes that influence EMS report availability showed the internal process time from fax report receipt to distribution significantly improved. This improvement reflected the change strategies that were all directed at improving the internal process.

EMS patient care reports are more efficiently processed and distributed in the ED due to change strategies implemented that targeted the ED's internal process of EMS report distribution. The external process responsible for transmitting EMS reports to the ED is the limiting factor that prevents consistent timely access of EMS reports by emergency physicians and will require dedicated improvement strategies.

\section{Problem}

Transition of care is a critical period for ensuring important clinical information is relayed among healthcare providers. Good handover has been shown to be associated with improvements in patient safety, continuity of patient care, and improved decision making.[1] Prehospital to hospital transition of care most frequently occurs between paramedics and emergency triage nurses. A verbal report is typically provided by paramedics and documented by triage nurses. Paramedics subsequently complete a patient care report which is submitted electronically.
The emergency medical system (EMS) patient care report often contains details of paramedic assessment and management that is not all captured in the nursing triage note. Sometimes the EMS report can be helpful to clarify on scene witness description of events, cardiac rhythm strips, or interventions by paramedics and subsequent clinical responses.

EMS patient care reports are often unavailable for review by emergency physicians and nurses. Some emergency departments do not have a system to distribute reports while patients are being assessed in the emergency department resulting in the reports not being available for any patients arriving by ambulance. Many 
emergency departments have a system to distribute reports but due to delays in processing the reports they are often unavailable to healthcare providers.

Two processes occur in the distribution of EMS patient care reports. The first is an external process to the emergency department (ED) that is influenced by the prehospital emergency medical system. Once paramedics complete their documentation on a mobile computer it is sent to a server which then transmits the report to the ED by fax. The second process is an internal process to the ED that requires clerical staff to distribute the fax report to accompany the patient chart.

There are many factors that contribute to delays in both the external and internal processes. After initial discussions with Toronto EMS and realizing the major system changes that would be required to improve the external process it was decided to focus on improving the internal process initially and to then re-engage Toronto EMS in hope of later improving the external process.

\section{Background}

Communication failures are a major cause of adverse events in healthcare. Strategies to improve exchange of information among healthcare professionals have been shown to mitigate risk to patients. Clinical handovers of patient care from one healthcare professional to another is vulnerable to the loss of important clinical information. A literature review of handover of patients arriving by ambulance to the ED identified that important information may be missed during clinical handover.[2]

Barriers to effective clinical handovers between paramedics and ED staff include a lack of common language or understanding between healthcare disciplines, inattention to handover and lack of active listening skills, variable quality and quantity of information exchanged during handover, lack of teamwork skills, the busy and complex working environment, and repetition of handover.[2]

In one study evaluating information loss in EMS handover of trauma patients only $72.9 \%$ of key prehospital data points verbally transmitted by paramedics were documented by the receiving hospital staff.[3] To counteract deficiencies in information retention during handover from prehospital to the hospital setting, one recommendation is for ED staff to have timely access to written ambulance reports to provide accurate and detailed information about the patient's initial presentation and EMS management.[2]

There is much literature on the use of electronic medical records to improve the process of clinical handovers. It is recognized that information technology plays an important role in achieving major gains in quality and patient safety including handovers.[4]

\section{Baseline measurement}

For six consecutive days during various shifts in the ED the percentage of EMS patient care reports available to emergency physicians at the time of initial patient assessment was determined.
This data is shown in figure 1 (before changes implemented). There was wide variation in the availability of EMS reports to the treating physician.

For four consecutive days during various ED shifts the time interval from patient transfer from EMS to ED stretcher until the EMS report was received by fax was measured for all patients arriving in the ED by ambulance. This data is shown in figure 2 (before changes implemented). Again there was wide variation in how long it took to receive the EMS report by fax with some outliers of over eight hours. Also for four consecutive days during various ED shifts the time from receiving the EMS fax report until distribution to patient charts was measured and is displayed in figure 3 (before changes implemented). Wide variation also exists for this internal process to the ED.

\section{Design}

During a focus group with ED clerical staff misconceptions related to EMS patient care reports were identified. Clerical staff thought the reports were not read by emergency physicians and nurses and that all necessary information was conveyed at the time of verbal handover from paramedic to triage nurse. Therefore correcting these misconceptions was important in order for clerical staff to place value on their duty of EMS report distribution.

It was identified that the ED at Sunnybrook Health Sciences Centre, Toronto, Canada received all fax EMS reports for every patient transferred to the hospital. Since Sunnybrook is a tertiary care hospital this included interhospital transfers and code ST-segment elevation myocardial infarction (STEMI) patients that bypassed the ED. For all faxes of EMS reports received in the ED, clerical staff had to first determine if the patient was an active ED patient. If a patient was not being managed in the ED at the time the report was received then their location within the hospital was determined and the reports were redirected by fax or intrahospital mail. This sometimes led to a delay in distributing EMS reports for active ED patients. We developed a new system to minimize ED involvement of EMS reports received for nonactive ED patients.

An electronic dashboard for ED patients arriving by ambulance was built to aid clerical staff in quickly identifying and locating such patients. The goal was to facilitate more efficient distribution of EMS reports.

Quality initiatives that decrease workload are more likely to achieve reliable performance and sustainability. Since ED clerical staff were being tasked with improving the efficiency of EMS report distribution one of their many job duties was identified for elimination to offset any perceived workload demand. Since the introduction of electronic patient charts for discharge summaries in Sunnybrook over 10 years ago, paper hospital charts are infrequently used. And yet hospital charts were retrieved from the health records department for most ED patients. This routine process of retrieving old charts for ED patients was stopped. Emergency physicians were initially informed of this proposed change and feedback encouraged to ensure there was no significant impact on patient care. 


\section{Strategy}

PDSA cycle 1 focused on educating ED clerical staff about the importance of EMS reports. Methods of education included an email to all clerical staff and a poster display listing the reasons why EMS reports are important. Much informal discussion also occurred with clerical staff to reinforce the message and to answer questions.

PDSA cycle 2 first consisted of meeting with the director of the hospital's health records department and requesting health records staff process and distribute EMS reports for nonactive ED patients. Subsequently a new process was developed to minimize ED clerical staff handling of EMS reports for nonactive ED patients. The process was eventually streamlined after feedback from clerical staff and determined that these EMS reports would be placed in an envelope and sent to the health records department each morning.

PDSA cycle 3 first consisted of building an electronic dashboard to make it easier for clerical staff to identify and locate ED patients that arrive by ambulance. Once the dashboard was built clerical staff were asked to use it and feedback was received to make modifications. The time was tracked to determine how long it took to process EMS reports. Ultimately the goal was to use the electronic dashboard to facilitate more efficient distribution of EMS reports.

PDSA cycle 4 eliminated the retrieval of old hospital charts and their distribution for ED patients. A minor change was made to the computer program used to register patients in the ED so that an automatic process requesting old hospital charts was ceased. Emergency physicians were informed of this change and the ability to phone to request a chart was maintained. This resulting decrease in workload for the health records department was used as leverage to facilitate agreement of the same department to process EMS reports for nonactive ED patients.

\section{Results}

Implementation of change strategies did not result in a significant improvement in the percentage of EMS reports available to emergency physicians at the time of initial patient assessment (figure 1). However, tracking both external and internal processes that influence EMS report availability showed that the internal process time from fax report receipt to distribution significantly improved (figures 2 and 3 ). This improvement reflected the change strategies that were all directed at improving the internal process.

The hospital's health records department tracked the number of old charts requested for ED patients after elimination of the automatic retrieval process. On average two charts were requested each month.

See supplementary file: ds6708.docx - "Pre and post outcome and process measurements (figures 1 to 3 )"

\section{Lessons and limitations}

Improvement in a process cannot be fully realized unless all stakeholders are engaged and part of the improvement process. Our aim of improving the percentage of EMS reports available to emergency physicians at the time of initial patient assessment did not occur because EMS stakeholders were absent from improvement planning and discussions.

By educating clerical staff about the importance of EMS reports on patient care they could place value on the task of distributing the reports. In doing so one is changing mindset and not just behaviour.

Undertaking a quality improvement initiative is an ideal time to look at opportunities to eliminate waste that may positively impact the quality initiative. It was identified that electronic health records minimized the utility of old patient charts in paper form.

A cost savings of the elimination of automatic retrieval of old charts was realized by the health records department which presented an opportunity to delegate the processing of EMS reports for nonactive ED patients to health records department staff.

\section{Conclusion}

EMS patient care reports are more efficiently processed and distributed in the ED due to change strategies implemented that targeted the ED's internal process of EMS report distribution. It is now evident that the external process responsible for transmitting EMS reports to the ED is the limiting factor that prevents consistent timely access of EMS reports by emergency physicians. After reviewing the steps involved in the external process it is likely that any significant improvement in this process will be gained by use of online transmission and elimination of fax transmission of the reports.

Although EMS reports may not be read on all patients arriving in the ED by ambulance, it is only by making all reports more efficiently available to emergency physicians that the necessity of a select number of reports will be ensured to optimize patient safety.

\section{References}

1. Wood K, Crouch R, Rowland E, Pope C. Clinical handovers between prehospial and hospital staff: literature review. Emerg Med J 2015;32(7):577-81.

2. Bost N, Crilly J, Wallis M, Patterson E, Chaboyer W. Clinical handover of patients arriving by ambulance to the emergency department - a literature review. Int Emerg Nurs 2010;18(4):210-20.

3. Carter AJ, Davis KA, Evans LV, Cone DC. Information loss in emergency medical services handover of trauma patients. Prehosp Emerg Care 2009;13(3):280-5.

4. Till A, Sall H, Wilkinson J.Safe Handover : Safe Patients The Electronic Handover System. BMJ Qual Improv Report 2014;2: doi:10.1136/bmjquality.u202926.w1359

\section{Declaration of interests}

Nothing to declare. 


\section{BMJ Quality Improvement Reports}

\section{Acknowledgements}

The authors would like to thank Ajibike Oyewumi, Darren Hefferon, Angie Jeffs and Jeffrey Tyberg for their support with this project.

\section{Ethical approval}

According to Sunnybrook Research Institute this project met the criteria for operational improvement and was exempt from research ethics review. 